\title{
3D Printing for Rapid Manufacturing: Study of Dimensional and Geometrical Accuracy
}

\author{
Hirpa G. Lemu and Safet Kurtovic \\ Department of Mechanical Engineering and Materials Technology \\ University of Stavanger, N-4036 Stavanger, Norway \\ Hirpa.g. lemu@uis.no
}

\begin{abstract}
D printing (3DP) is one of the innovative developments in rapid prototyping (RP) technology. The goal of the initial inception and progress of the technology was to assist the product development phase of product design and manufacturing. The technology has played an important role in educating product design and 3D modeling because it helps students/designer to visualize their design idea, to enhance their creative design process and enables them to touch and feel the result of their innovative work. This paper presents the results of the study done on the in-built potentials and limitations of 3DP technology when used for rapid manufacturing purposes.
\end{abstract}

Keywords: 3D printing, rapid prototyping \& manufacturing, printing accuracy.

\section{Introduction}

Rapid prototyping (RP) technologies are nowadays widely applied for production of parts that are based on additive fabrication principles. The technology integrates key disciplines and sets a different approach to the traditional procedure from design to manufacturing where 3D physical model of any shape directly from a Computer aided design (CAD) model is built layer by layer. As one of the leading RP technologies, the inception and development of 3DP technology has highly contributed in the product development phase of a product. Among others, the technology provides a unique opportunity to control the material composition [1-3] of the product by jetting different powder-based materials from different nozzles. The technology is playing an important role in educating product design and 3D modeling because it helps students/designers to visualize their design idea and to enhance their creative design process. It stimulates innovative work because it enables designers to touch and feel the result of their idea. It simplifies communication between different actors of a product, even with nontechnical ones.

Further, it enables to develop prototypes at a comparatively high speed and low cost. The development of many other capabilities and techniques such as selective use of different materials, suitable post treatment (PT) techniques and color printing capabilities have opened many and diverse fields of applications. 


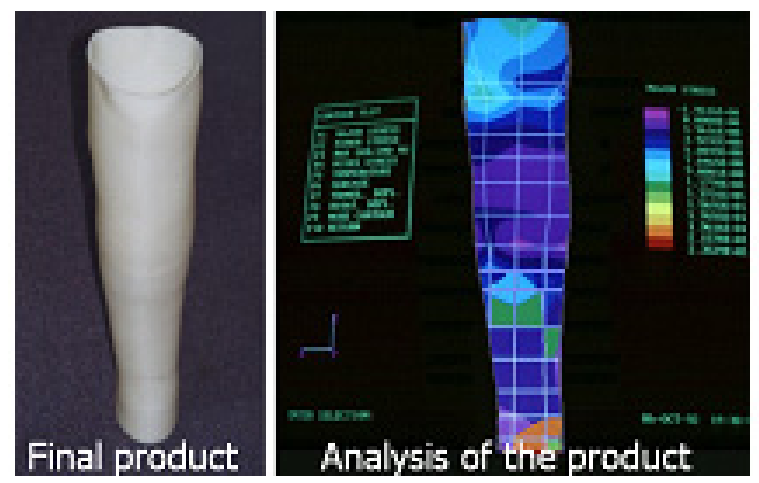

Fig. 1. Example of integrating 3DP with finite element analysis, adapted from [4]

These include pattern making, design aids for tooling equipment, anatomical modeling and prototyping of human organs and implants, reconstructive surgery aids and others. Emerging new applications are also enabling 3DP machines to satisfy further scientific and engineering needs such as molecular modeling and the presentation of the results of finite element analysis (FEA). The biomedical area represents a typical example where integration of 3D modeling, 3D printing and FEA play a significant role in the future (Fig. 1). As physical testing of prostheses is demanding, full FEA in this area can open opportunities to apply the gained knowledge on new designs. In recent years, the application of RP technology in medical area has grown and a new discipline, known as medical rapid prototyping (MRP) is in the making [5]. The advance in this direction will involve manufacture of accurate $3 \mathrm{D}$ physical models of human anatomy derived from medical image data.

Another potential area is integration of advanced 3D modeling technologies with thermal and flow analysis through computational fluid dynamics (CFD) simulation. This enables application of advanced solid modeling techniques such as NURBS to improve the shape for aerodynamic performance. The prototype can easily be built in 3DP, without tooling, and digital or physical testing enables integration of advanced 3D modeling, 3D printing as well as digital and physical testing. Some researchers indicate, however, that this technology as a rapid manufacturing tool remains at present more of a goal than reality for the industry [6].

Based on the huge potential that 3DP technology can play in the future to realize true RP\&M, many 3DP products with varying capabilities are now available on the market. One of the most future oriented 3DP technologies is marketed by ZCorporation (Z-corp.) that is based on MIT's ((Massachusetts Institute of Technology) ink jet technology. This 3DP variant is classified as a typical "concept modeler", a low-end system, and represents the fastest RP process. It is rapidly spreading worldwide and it has become the third most widely used layered manufacturing equipment within three years of its early market life [7]. 
On the other hand, the availability of diverse technologies in 3D printing has created certain level of challenge for the user because of varying capabilities and limitations to a particular need. Literatures published within the last decade indicate that complexity of part geometry, material used in the prototyping model, compatibility with 3D CAD models and other technical aspects still need in-depth study. The major problems focused in those recent researches include: accuracy and limited availability of materials [8], porosity [9], and surface finish [10]. These limitations can result in limited range of mechanical properties. Additional research issues of 3DP at current stage involve part size and profile (including thickness), compatibility with 3D CAD models and other computer aided tools, application ranges and customer satisfaction.

Our in-house experience with the use of Z510, one of the latest machines from Zcorp., also indicates the need for further research to clearly identify the potentials and limitations of this technology. The study partially presented in this paper is aimed to build a full capability profile of 3DP technology including dimensional and geometrical accuracy, data transfer compatibility, surface roughness, build time and strength (in terms of wall thickness) of this technology. In the end, the accumulated knowledge from such studies will contribute in classification of the 3D printing qualities in accordance with international standards.

\section{Brief Description of the Z510 3DP and the Printing Process}

The study reported in this article is based on the capabilities and limitations of Z510 3D printer (3DP). This machine was selected because the CAE laboratory of the University of Stavanger owns the machine.

The part building method using RP technologies has a series of important features or procedures (Fig. 2) that are in general almost identical for all RP machines. All of the technologies require input of a solid model from a 3D CAD system, usually as slices. The designed model from a CAD system is then tessellated and exported to a suitable file format. With its development roots in the 1960s, STL-format is the current industry standard for facetted models. The STL file represents the model using information about the coordinates and outward surface normal of triangles. At this phase, the technology integrates $\mathrm{CAD}$ and CAM (computer-aided manufacturing) avoiding elaborate process planning and machine set up activities.

When the part model is ready in the printing machine, two important processes take place upon pressing the start button: (1) the software calculates how the layers look like for the model and (2) printing starts when five layers are calculated. This is one of the features of 3DP that makes it best in terms of the building speed. The manufacturers claim that 3DP from Z-corp is $5-10$ times faster than other RP technologies. The machine uses a default layer thickness of $0.1016 \mathrm{~mm}$; if not a particular layer thickness is selected by the operator. 


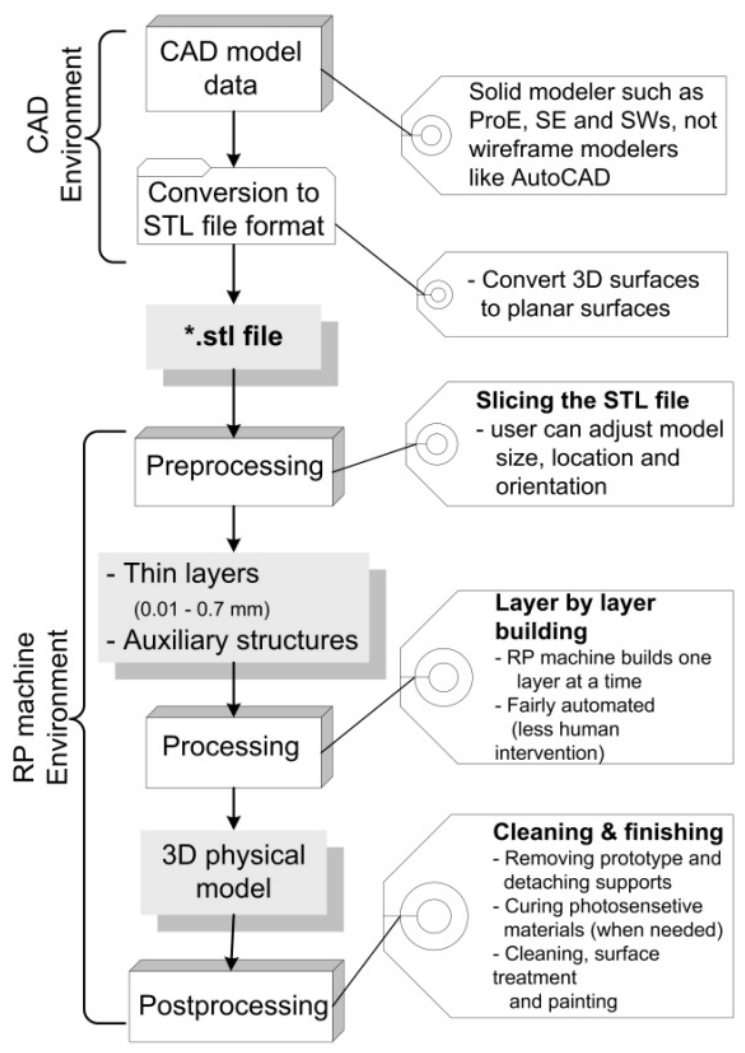

Fig. 2. Common features or procedures of RP technologies
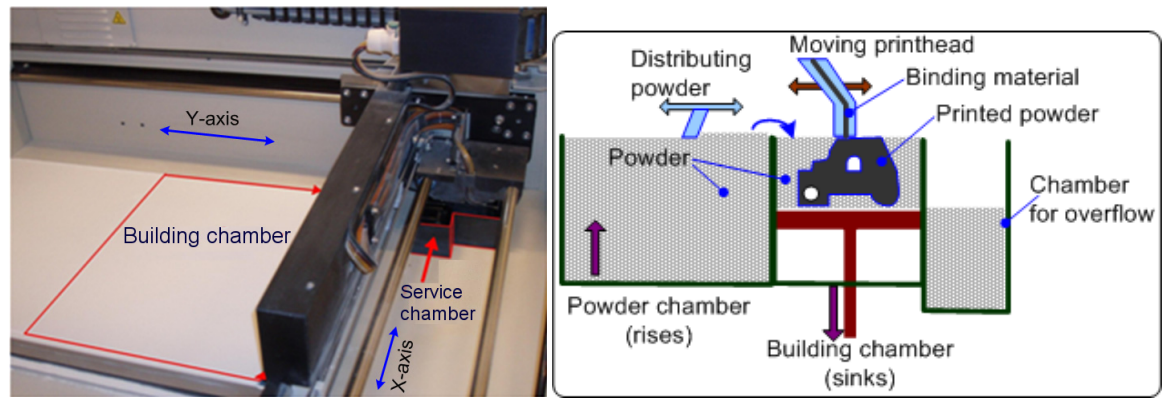

Fig. 3. Coordinate system definitions and components of the part building area in 3DP

Figure 3 shows the definition of the coordinate systems and the important components in the part building area. By convention, the data slicing takes place in the $\mathrm{X}-\mathrm{Y}$ plane and the part is built in the $\mathrm{Z}$ direction. The movement along the $\mathrm{X}$-axis is designated as the slow speed axis and its task is to lay the binding materials. The Y-axis, on the contrary is a high speed axis. These two movements cover the $2 \mathrm{D}$ geometry of the 
development area of the model. The volume is then defined by the vertical movement of the building chamber corresponding to the layer thickness.

As illustrated in Figure 3 (right), the printer performs 5 different operations in the process of printing (adding a layer of powder).

1. Lowering the powder chamber

2. Moving the printhead back the powder chamber

3. Lifting the powder chamber

4. Lowering the building chamber and

5. Distributing the powder from the powder chamber to the building chamber.

Some 3DP machines need warming up to $38^{\circ} \mathrm{C}$ so that the binding material sets quickly. Latest machines, such as Z510 - the one available in our laboratory, however, do not need this. It warms up after the model is developed so that it can cure.

\section{$3 \quad$ Tests and Materials}

There are many possible sources of surface inaccuracy of products produced by 3D printing process. For instance, error in data exchange process at preprocessing, part positioning and layer thickness at processing, and finishing works or post print operations at postprocessing phase can be mentioned. Closer observations show that achieving accuracy as specified by manufacturers is not always an easy task. Based on the experience of the existing limitations and supported by indications from the literature study, some test cases were identified. This paper presents the tests done on the following selected cases:

- influence of file transfer formats on part accuracy,

- achievable accuracies such as flatness and surface finish and

- minimum wall thickness.

Z510 uses mainly four powder materials. The main powder material used in the tests conducted as part of this study is ZP 131, high performance composite powder that is material of choice for color printing. The other three materials, i.e., elastomeric material, ZP14 Investment casting material and ZCast 501 Direct metal casting material, are considered as special material types for prototyping parts with specific need.

\section{Discussion of Test Results}

\subsection{Influence of File Transfer Formats on Part Accuracy}

All RP machines by default use STL (standard template library or streriolitography file format). Observing inaccuracies compared with manufacturer specifications, a comparative study between the data transfer formats was proposed. The rationale to make this test is also that STL file format is an old file transfer format from 1970's 
that has been used to describe straight lines and planar surfaces. The format cannot represent curved surfaces exactly. Z510 supports four file formats: STL (standard), VRML, PLY and 3DS. The CAD system used for 3D modeling of the part (Solid Edge) on the other hand supports, among other neutral file formats, the STL and VRML file formats that are compatible with 3DP. For this test, a 3D model was developed (Fig. 4(a)) with intentionally introduced features such as cylindrical and prismatic holes with different size and curved external edges. The same 3D model was transferred to 3DP software using the two file formats. The printed prototypes are shown in Fig. 4(b) and (c).

Geometrical Deviation: The difference in geometrical deviation between the two printed models is clearly visible with a naked eye. The inaccuracy in the cylindrical (hole) surface, i.e. roundness and the curved edge indicates that the models built using STL file transfer are less accurate than those built from VRML files. The VRML file format without exception produces circular features with better accuracy because it approximates curved edges with finer chords resulting in better circularity or profile form accuracy. The accuracy improves when the radius of the curve/circle decreases.

Dimensional Deviation: Dimension of models built from each file format were measured and compared with the original dimensions in the CAD model. The results of selected dimensions are shown in Fig. 5 together with tabulated dimensions. Apart from a couple of exceptions, the results show that interior dimensions shrink, while exterior dimensions expand in all directions. This indicates that the material volume increases in both cases. The ZPrint software has a function known as anisotropic scaling with a scaling factor between 0.8 and 1.2 that is intended to compensate for this material volume deviation particularly in experience based generalized scaling. Otherwise, the final product should undergo post print operations such as grinding, cutting or drilling

Though STL file format is the default file transfer format for 3DP and many other data exchange processes, this study reveals that it is not as good as the VRML format in terms of dimensional and geometrical accuracy. This obvious drawback puts a question mark on the possible use of this technology for rapid manufacturing purposes.

(b)

(a)

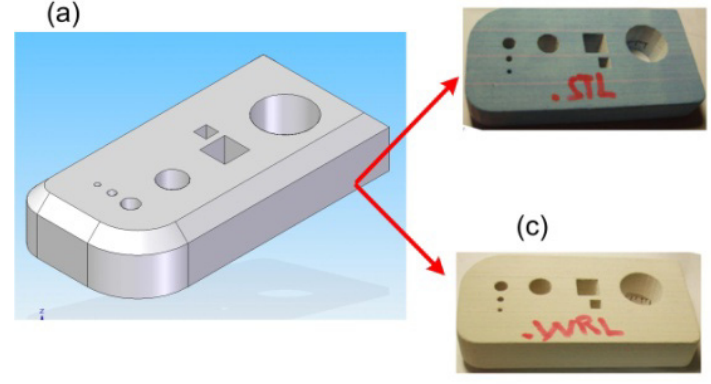

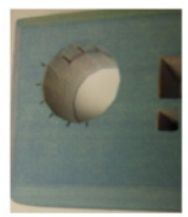
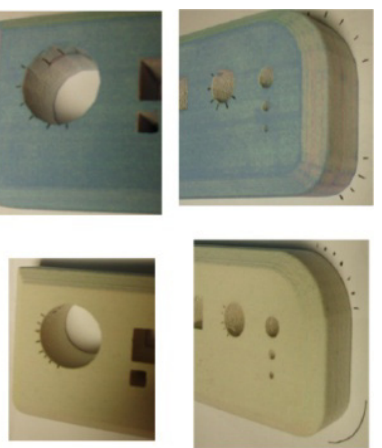

Fig. 4. Test samples: (a) original CAD model (b) part built from STL file as input (c) part built from VRML as input 


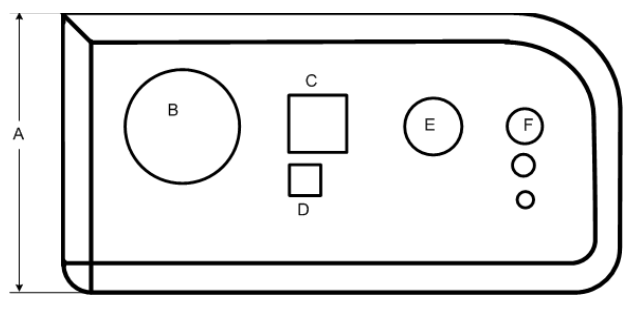

\begin{tabular}{|c|c|c|c|}
\hline Dimension & $\begin{array}{c}\text { CAD } \\
\text { model }\end{array}$ & $\begin{array}{c}\text { STL } \\
\text { model }\end{array}$ & $\begin{array}{c}\text { VRML } \\
\text { model }\end{array}$ \\
\hline $\mathrm{A}$ & 50.00 & 50.15 & 50.10 \\
\hline $\mathrm{B}$ & $\varnothing 20.00$ & $\varnothing 19.55$ & $\emptyset 19.60$ \\
\hline $\mathrm{C}$ & 10.00 & 9.95 & 9.80 \\
\hline $\mathrm{D}$ & 5.00 & 5.05 & 5.00 \\
\hline $\mathrm{E}$ & $\varnothing 10.00$ & $\varnothing 9.70$ & $\varnothing 9.90$ \\
\hline $\mathrm{F}$ & $\varnothing 6.00$ & $\varnothing 5.65$ & $\varnothing 5.70$ \\
\hline
\end{tabular}

Fig. 5. Comparison of dimensional deviations

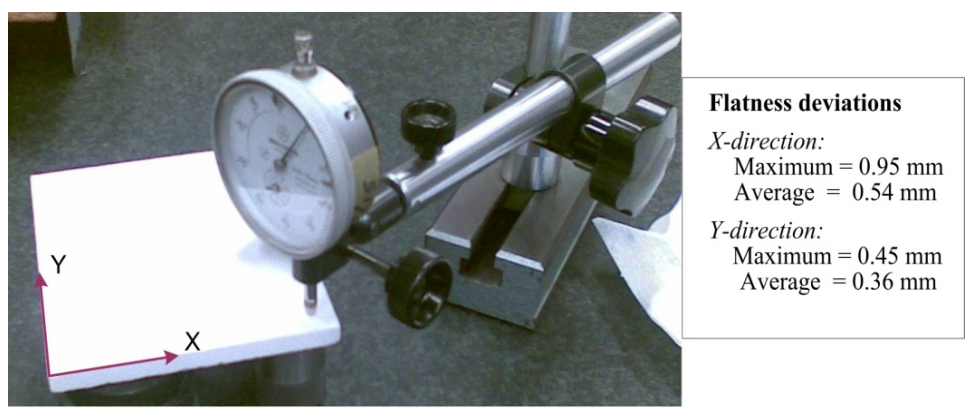

Fig. 6. Test setup and measured and measured values for flatness deviations

\subsection{Test for Achievable Surface Flatness}

As mentioned earlier, printing in 3DP is done without support structures, i.e. the under laying powder supports the built model. This, on the other hand, influences the accuracy on surface flatness. Though the printing temperature is not so high to create significant thermal deformations, moderately good flatness requirement for relatively thin features may not be expected.

For this test, a simple thin plate of size $100 \times 120 \times 8 \mathrm{~mm}$ was printed and the flatness accuracy was measured using a dial indicator (Fig. 6). The measured deviations show that the flatness error is highest in the X-direction, max. 0.95 and average $0.54 \mathrm{~mm}$, while the deviation in Y-direction is max. 0.45 and average 0.36 $\mathrm{mm}$. According to ISO 2768-2 the general geometrical tolerance for flatness recommended for this size is: H-class (fine) $=0.2 \mathrm{~mm}, \mathrm{~K}$-class (medium) $=0.4 \mathrm{~mm}$ and L-class $($ rough $)=0.8 \mathrm{~mm}$. This indicates that the 3DP process achieves a flatness accuracy that is almost equivalent to that of machining parts from steel materials.

\subsection{Test for Achievable Surface Finish}

Though Z-corp [11] claims that their 3DP machines are known to have ultra-smooth surface quality compared with other RP technologies, reports from some published data $[12,13]$ indicate as this is not the case. Thus, in addition to developing better understanding of the achievable surface quality in 3DP in general and using Z510 in particular, this test was intended to study the influence of post treatment such as hardening and polishing on the surface quality. Further, a surface quality comparison 
between a surface normal to the building direction (the XY plane) and the surface parallel with the part building direction (XZ plane) was done.

Two parts - untreated part and a post-treated (hardened) part were tested. One face of each sample was polished while the rest were not. Among others, the following materials and equipment were used: polishing machine (Knuth-Rotor 2), epoxy (XD4360), hardener (XD4361) and Mitotoyo surfacetest-20.1.

The Mitotoyo surface test apparatus gives surface roughness qualities in three parameter values: $R_{A}, R_{Z}$ and $R_{T}$. In order to study the influence of the building orientation and the post treatment on the surface roughness, measurements were done on both as printed and post treated surfaces. The measurements were taken on the surface parallel to the building direction (on XZ-plane) and on one of the surfaces normal to the building direction (on XY-plane).

The test results for these samples are given in Table 1. Apart from few exceptions, the XZ-plane has the highest roughness level as expected. This is due to the stair-steps formed by the layer thickness while building. This roughness is expected to increase for curved edges and surfaces. The results also show that post treatment operations like hardening reduce the possibility to improve the surface quality by polishing.

Table 1. Measured surface roughness values $(\mathrm{Ra})$ in $[\mu \mathrm{m}]$

\begin{tabular}{rcccccccc}
\hline & \multicolumn{4}{c}{ As printed } & \multicolumn{3}{c}{ Hardened } \\
\cline { 2 - 9 } Unpolished & \multicolumn{2}{c}{ Polished } & \multicolumn{2}{c}{ Unpolished } & \multicolumn{2}{c}{ Polished } \\
Plane & XY & XZ & XY & XZ & XY & XZ & XY & XZ \\
\hline Sample & A1 & B1 & C1 & D1 & E1 & F1 & G1 & H1 \\
Single measurement & 7.76 & 17.74 & 13.99 & 9.73 & 9.84 & 21.63 & 0.34 & 8.7 \\
Average roughness & 7.82 & 16.05 & 13.40 & 11.25 & 9.73 & 20.95 & 2.22 & 6.15 \\
\hline
\end{tabular}

\subsection{Minimum Printable Wall Thickness}

This test represents the most important part of this project where understanding the lowest possible part thickness that can be safely built is sought. This is especially important in cases of down scaling a prototype. The study was intended to reveal the possible risks of damaging the prototype under the following steps:

1. Processing (part building)

6. Withdrawal from the building chamber

7. Depowdering and

8. Post treatment of the prototype.

Two test models were drawn with features of different thickness (down to $1 \mathrm{~mm}$ ) and located in the building chamber with different orientations as shown in Fig. 7 (L). The orientations were intended to study the significance of having the part against the $\mathrm{XY}-\mathrm{YZ}$ walls and XY-XZ walls. 

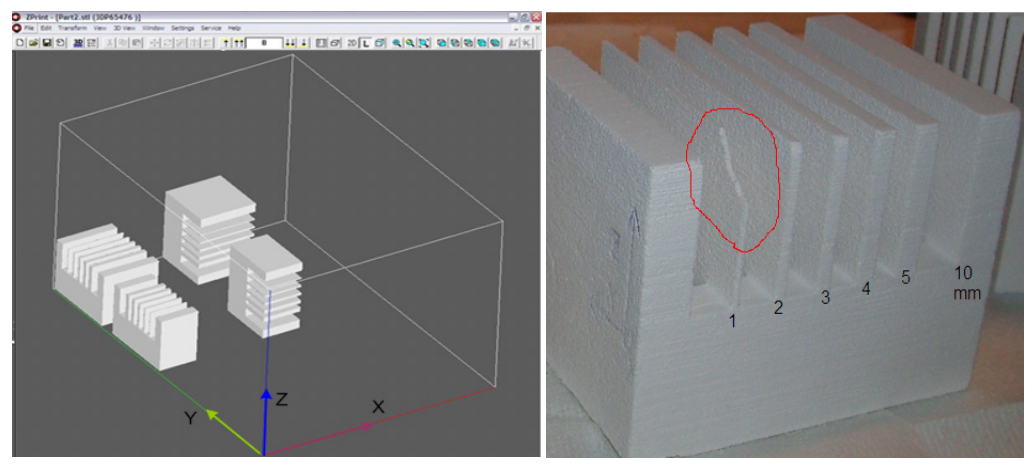

Fig. 7. Part orientation in building chamber (L) and an example of a broken part (R) under test

All of the samples survived the first test (processing stage) while removal from the building chamber was not error free, at least needs extreme care for thin-walled parts. One example of a broken part under this postprocessing stage is shown in Fig. 7 (R). The general conclusion drawn from this test is that orientation of thin-walled parts is very important. With no doubt, resting the face of a thin-walled feature against the XZ-plane increases the risk of damaging the prototype while withdrawing from the building chamber. The study also shows that orienting the weakest geometry against the XY-plane avoids this problem, for instance buckling including due to the part's own weight. This consideration, however, contradicts with the orientation of a part in the building chamber with respect to an optimum printing time. In other word, the orientation that secures good part strength is not always the optimum orientation.

A Case Study: a typical wall thickness problem is of high concern when parts are downscaled to the size that can be printed by the 3DP (maximum $350 \mathrm{~mm}$ ). Several cases were studied including a bobsled that was modeled in the CAD tool and printed. The model with maximum length of $3200 \mathrm{~mm}$ was downscaled to $10 \%$ size and this represents an obvious challenge for the wall thickness. As illustrated in Fig. 8, several weak points were observed that needed extreme care during postprocessing. Repeated trials indicate that experience in handling and positioning avoids some of the observed failures.

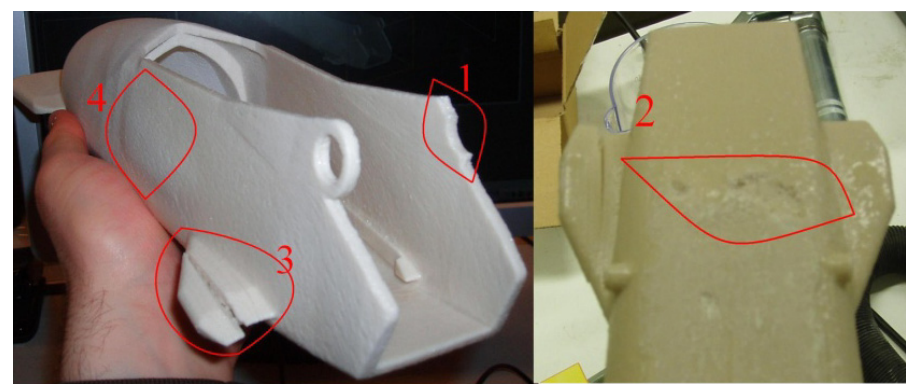

Fig. 8. Examples of inaccuracies and failures on printed bobsled 


\section{Conclusion}

Initiated by in-house experience with the use of 3DP, the study on dimensional and geometrical accuracy of Z510 from Z-corp. has been reported in this article. The investigated limitations are of course not limited to this specific 3DP machine. Experimental tests on the influence of file transfer formats on part accuracy, dimensional and geometrical deviations and minimum wall thickness were studied. The research findings indicate that both dimensional and geometrical deviations take place on printed parts and the size of the deviations depends on the type of file transfer format. This study reveals also that STL format in general is not as good as VRML format. While the achievable flatness accuracy of 3DP in general is comparably as good as machining operations, the achievable surface roughness depends on the layer thickness and geometric form. The wall thickness is an important factor to be considered particularly when the part is to be downscaled. The study results indicate that manageable minimum wall thickness depends on several factors including the positioning of the part in the chamber.

Acknowledgement. The technical support provided by Y. A. Amith is appreciated.

\section{References}

1. Hopkinson, N., Hague, R., Dickens, P.: Rapid Manufacturing: An Industrial Revolution for the Digital Age. Wiley, Chichester (2006)

2. Jackson, T.R., Liu, H., Patrikalakis, N.M., Sachs, E.M., Kima, M.J.: Modeling and Designing Functionally Graded Material Components for Fabrication with Local Composition Control. Mater. Design 20(2-3), 63-75 (1999)

3. Cho, W., Sachs, E.M., Patrikalakis, N.M., Troxel, D.E.: A Dithering Algorithm for Local Composition Control with Three-dimensional Printing. Comput.-Aided Des. 355(9), 851-867 (2003)

4. http://rehabtech. eng.monash. edu.au/cadcam/INFO/present.htm (last access: April 18, 2012)

5. Hieu, L.C., Zlatov, N., Vander Sloten, J., Bohez, E., Khanh, L., Binh, P.H., Oris, P., Toshev, Y.: Medical Rapid Prototyping Applications and Methods. Assembly Automation 25(4), 284-292 (2005)

6. Dimitrov, D., Schreve, K., De Beer, N.: Advances in Three Dimensional Printing - State of the Art and Future Perspectives. Rapid Proto J. 12(3), 136-147 (2006)

7. Bak, D.: Rapid Prototyping or Rapid Production? 3D Printing Processes move Industry Towards the Latter. Assembly Automation 23(4), 340-345 (2003)

8. Levy, G.N., Schindel, R., Kruth, J.P.: Rapid Manufacturing and Rapid tooling with Layer Manufacturing ( $\mathrm{lm})$ Technologies, State of the Art and Future Perspectives. Annals of the CIRP 52(2) (2003)

9. Dimitrov, D., Van Wijck, W., Schreve, K., De Beer, N.: Investigating the Achievable Accuracy of Three Dimensional Printing. Rapid Proto J. 12(1), 42-52 (2006)

10. Karapatis, N.P., Van Griethuysen, J.-P.S., Glardon, R.: Direct Rapid Tooling: A Review of Current Research. Rapid Proto J. 4(2), 77-89 (1998)

11. (March 07, 2011), http://www.zcorp.com/en/Products/Rapid-Prototy ping-Machines/spage.aspx

12. Stankiewicz, M., et al.: The Scope of Application of Incremental Rapid Prototyping Methods in Foundry Engineering. Arch. Foundry Eng. 10, 405-410 (2010)

13. Frank, W.L.: Rapid Prototyping and Engineering Applications, A Toolbox for Prototype Development. Taylor \& Francis Group, Boca Raton (2008) 